\title{
PIECEWISE MONOTONE POLYNOMIAL APPROXIMATION
}

\author{
BY
}

\section{J. NEWMAN, ELI PASSOW AND LOUIS RAYMON}

\begin{abstract}
Given a real function $f$ satisfying a Lipschitz condition of order 1 on $[a, b]$, there exists a sequence of approximating polynomials $\{P\}$ such that the sequence $E_{n}=\left\|P_{n}-f\right\|$ (sup norm) has order of magnitude $1 / n$ (D. Jackson). We investigate the possibility of selecting polynomials $P$ having the same local monotonicity as $f$ without affecting the order of magnitude ${ }^{n}$ of the error. In particular, we establish that if $f$ has a finite number of maxima and minima on $[a, b]$ and $S$ is a closed subset of $[a, b]$ not containing any of the extreme points of $f$, then there is a sequence of polynomials $P_{n}$ such that $E_{n}$ has order of magnitude $1 / n$ and such that for $n$ sufficiently large ${ }^{n} P_{n}$ and $f$ have the same monotonicity at each point of $S$. The methods are classical.
\end{abstract}

Let $C[a, b]=\{f: f$ continuous on $[a, b]\}$, with $\|f\|=\max _{x \in[a, b]}|f(x)| . \omega(f, b)=$ $\omega(b)$ will denote the modulus of continuity of $f . P_{n}$ and $T_{n}$ are, respectively, the spaces of algebraic and trigonometric polynomials of degree less than or equal to $n$. $C, C_{1}, C_{2}, \ldots$ denote absolute positive constants.

$f \in C[a, b]$ will be called piecewise monotone if it has a finite number of maxima and minima on $[a, b] . \quad a, b$ and the local maxima and minima of $f$ will be called the peaks of $f$. It follows from a result proved independently by $W$. Wolibner [6] and S. W. Young [7] that if $f$ is a piecewise monotone function on $[a, b]$ and $\epsilon>0$, then there exists an algebraic polynomial $p$ which increases and decreases simultaneously with $f$ on $[a, b]$ and satisfies $\|f-p\|<\epsilon$. We are concerned here with the accuracy of this type of approximation as a function of the degree of the approximating polynomial. Jackson's classic theorem $[2, \mathrm{p} .56]$ states that for any function $f \in C[a, b]$ there exists $p \in P_{n}$ such that $\|f-p\|<$ $C_{1} \omega(f, 1 / n)$. O. Shisha [5], J. Roulier [4], and G. G. Lorentz and K. L. Zeller [1] have obtained results on the accuracy of the approximation of an increasing function by increasing polynomials of degree less than or equal to $n$. Lorentz and Zeller have shown that for each increasing function $f$ on $[a, b]$ and for each $n>0$ there exists an increasing $p \in P_{n}$ such that $\|f-p\|<C_{2} \omega(f, 1 / n)$. They have also shown that for any function $f$, increasing on $[-\pi, 0]$ and even on $[-\pi, \pi]$, there exists an even polynomial $t \in T_{n}$, increasing on $[-\pi, 0]$, which satisfies $\|f-t\|<C_{3} \omega(f, 1 / n)$. Our main results are Theorem 3 and Theorem 4

Received by the editors September 8, 1971.

AMS (MOS) subject classifications(1970). Primary 41 A25, 41A10, $42 \mathrm{~A} 08$.

Key words and phrases. Monotone approximation, piecewise monotone approximation, Jackson kernel, Jackson's Theorem. 
in which we extend, in some sense, the result of Lorentz and Zeller to piecewise monotone functions.

We will let $J_{n}(x)$ be the Jackson kernel; i.e.,

$$
J_{n}(x)=\frac{1}{\lambda_{n}}\left(\frac{\sin n x / 2}{\sin x / 2}\right)^{4}, \text { where } \lambda_{n}=\int_{-\pi}^{\pi}\left(\frac{\sin n x / 2}{\sin x / 2}\right)^{4} d x
$$

It is known [2, p. 54] that $n^{3} \leq \lambda_{n} \leq 2 \pi n^{3}$.

Lemma. $J_{n}(x)$ bas the following properties:

(i) If $n$ is even, $0 \leq \delta \leq \pi$, then

$$
\int_{0}^{\delta} J_{n}(x) d x \geq \int_{\pi-\delta}^{\pi} J_{n}(x) d x .
$$

(ii) If $0<\delta \leq \pi / 2$, then

$$
\int_{\delta}^{\pi} J_{n}(x) d x \leq C_{4} / n^{3} \delta^{3} .
$$

Proof. (i) Case 1. $0 \leq \delta \leq \pi / 2$.

Since $J_{n}(x)$ is an even periodic function,

$$
\int_{\pi-\delta}^{\pi} J_{n}(x) d x=\int_{\pi}^{\pi+\delta} J_{n}(x) d x .
$$

Thus,

$$
\int_{0}^{\delta} J_{n}(x) d x-\int_{\pi}^{\pi+\delta} J_{n}(x) d x=\int_{0}^{\delta}\left[J_{n}(x)-J_{n}(x+\pi)\right] d x
$$

Now, when $n$ is even,

$$
J_{n}(x)-J_{n}(x+\pi)=\frac{1}{\lambda_{n}}\left[\left(\frac{\sin n x / 2}{\sin x / 2}\right)^{4}-\left(\frac{\sin n x / 2}{\cos x / 2}\right)^{4}\right] .
$$

Since $(\tan x / 2)^{4} \leq 1$ for $0 \leq x \leq \pi / 2$, the integrand in (3) is nonnegative, and, hence, (3) is nonnegative for $0 \leq \delta \leq \pi / 2$.

Case 2. $\pi / 2 \leq \delta \leq \pi$.

Here

$$
\int_{0}^{\delta} J_{n}(x) d x-\int_{\pi-\delta}^{\pi} J_{n}(x) d x=\int_{0}^{\pi-\delta} J_{n}(x) d x-\int_{\delta}^{\pi} J_{n}(x) d x,
$$

and the result follows from Case 1.

$$
\int_{\delta}^{\pi} J_{n}(x) d x \leq \frac{1}{n^{3}} \int_{\delta}^{\pi}\left(\frac{\sin n x / 2}{\sin x / 2}\right)^{4} d x \leq \frac{1}{n^{3}} \int_{\delta}^{\pi} \frac{d x}{(\sin x / 2)^{4}} .
$$

Now $\sin (x / 2) \geq x / \pi$ for $0 \leq x \leq \pi$. Therefore 


$$
\int_{\delta}^{\pi} J_{n}(x) d x \leq \frac{\pi^{4}}{n^{3}} \int_{\delta}^{\pi} \frac{d x}{x^{4}}=\frac{\pi^{4}}{3 n^{3}}\left[\frac{1}{\delta^{3}}-\frac{1}{\pi^{3}}\right] \leq \frac{C_{4}}{n^{3} \delta^{3}},
$$

and the lemma is established.

Theorem 1. Let $r(x)=|x|,-\pi \leq x \leq \pi$, and be defined periodically by $r(x+2 k \pi)=r(x)$ for $k= \pm 1, \pm 2, \ldots$. Then there exisis $t \in T_{n}$ such that

$$
\max _{-\pi \leq x \leq \pi}|t(x)-r(x)|<C_{5} / n
$$

(ii) $t(x)$ decreases on $[-\pi, 0]$ and increases on $[0, \pi]$.

(iii) $t^{\prime}(x)$ increases on $[-\pi / 2, \pi / 2]$.

(iv) If $0<\delta \leq \pi / 2$, then for all $x \in[\delta, \pi-\delta]$

$$
1-C_{6} / n^{3} \delta^{3} \leq t^{\prime}(x) \leq 1,
$$

and for all $x \in(-\pi+\delta,-\delta),-1 \leq t^{\prime}(x) \leq-1+C_{6} / n^{3} \delta^{3}$.

(v) $\int_{-\pi}^{\pi}|d[t(x)-r(x)]| \leq 4 C_{5} / n$

Proof. For $n$ even let

$$
t(x)=\int_{-\pi}^{\pi} J_{n}(u) r(x-u) d u .
$$

By Jackson's Theorem, $t(x)$ satisfies (i). By periodicity,

$$
t(x)=\int_{x-\pi}^{x+\pi} J_{n}(u) r(x-u) d u=\int_{x-\pi}^{x} J_{n}(u)(x-u) d u-\int_{x}^{x+\pi} J_{n}(u)(x-u) d u .
$$

Hence

$$
\begin{aligned}
t^{\prime}(x) & =\int_{x-\pi}^{x} J_{n}(u) d u-\pi J_{n}(x-\pi)-\int_{x}^{x+\pi} J_{n}(u) d u+\pi J_{n}(x+\pi) \\
& =\int_{x-\pi}^{x} J_{n}(u) d u-\int_{x}^{x+\pi} J_{n}(u) d u .
\end{aligned}
$$

Let $x \in[0, \pi]$. By the periodicity and evenness of $J_{n}(u)$ we then get

$$
t^{\prime}(x)=2 \int_{0}^{x} J_{n}(u) d u-2 \int_{\pi-x}^{\pi} J_{n}(u) d u .
$$

Applying (1), $t^{\prime}(x) \geq 0$. By a similar argument $t^{\prime}(x) \leq 0$ for $-\pi \leq x<0$, proving (ii) for $n$ even. For $n$ odd choose

$$
t(x)=\int_{-\pi}^{\pi} J_{n-1}(u) r(x-u) d u
$$

and the proof follows as above. (i) and (ii) are established.

Proof of (iii). Differentiating in (7),

$$
t^{\prime \prime}(x)=J_{n}(x)-J_{n}(x-\pi)-J_{n}(x+\pi)+J_{n}(x)=2\left[J_{n}(x)-J_{n}(x+\pi)\right] .
$$

This quantity is nonnegative for $0 \leq x \leq \pi / 2$, as shown in the proof of part (i) 
of the Lemma. Then $t^{\prime \prime}(x) \geq 0$ for $-\pi / 2 \leq x \leq \pi / 2$ by evenness and periodicity of $J_{n}(x)$.

Proof of (iv). Let $x \in[\delta, \pi-\delta]$. We use (7) to obtain the desired estimates. Since $\int_{-\pi}^{\pi} J_{n}(u) d u=1$ and $J_{n}(u) \geq 0, t^{\prime}(x) \leq 1$. Now

$$
t^{\prime}(x) \geq \int_{-\delta}^{\delta} J_{n}(u) d u-\int_{\delta}^{2 \pi-\delta} J_{n}(u) d u
$$

which, by (2), gives

$$
t^{\prime}(x) \geq 1-2 C_{4} / n^{3} \delta^{3}-2 C_{4} / n^{3} \delta^{3}=1-C_{6} / n^{3} \delta^{3} .
$$

The estimates for $x \in(-\pi+\delta,-\delta)$ are similarly obtained.

Proof of (v). If $b(x)=t(x)-r(x)$, then $b^{\prime}(x)$ is $\leq 0$ on $[0, \pi]$ by (5). Hence the total variation of $b(x)$ on $[0, \pi]$ is bounded by

$$
2 \max _{0 \leq x \leq \pi}|t(x)-r(x)| \leq \frac{2 C_{5}}{n}
$$

by (4). Similarly the total variation of $b(x)$ on $[-\pi, 0]$ is bounded by $2 C_{5} / n$. Since $\int_{-\pi}^{\pi}|d b(x)|$ is equal to the total variation of $b(x)$ on $[-\pi, \pi]$, (v) is proved.

Definition. Let $f$ be piecewise monotone on $[a, b]$ with peaks at $a=x_{1}<$ $x_{2}<\ldots<x_{m}=b$. A sequence of polynomials $\left\{p_{n}\right\}$ is said to be comonotone with $f$ on $[a, b]$ if, for $n$ sufficiently large, $p_{n}$ increases and decreases simultaneously with $f$ on $[a, b] .\left\{p_{n}\right\}$ is said to be nearly comonotone with $f$ on $[a, b]$ if, for every $\epsilon$ satisfying $0<\epsilon<1 / 2 \min _{i}\left(x_{i+1}-x_{i}\right), p_{n}$ is comonotone with $f$ on $\left[x_{i}+\epsilon\right.$, $\left.x_{i+1}-\epsilon\right], i=1,2, \ldots, m-1$.

Using this terminology, the sequence of polynomials defined by (6) and (8) is comonotone with $r(x)$ on $[-\pi, \pi]$.

Definition. Let $a=y_{0}<y_{1}<\ldots<y_{k}=b$ and let $L \in C[a, b]$ be linear on $\left[y_{j}, y_{j+1}\right], j=0,1, \ldots, k-1$. Then $L$ will be called piecewise linear on $[a, b]$ and $y_{0}, y_{1}, \ldots, y_{k}$ will be called the nodes of $L$. Let $S_{j}$ be the slope of $L$ on $\left[y_{j}, y_{j+1}\right]$; we let $M(L)=\max _{j}\left|S_{j}\right|$ and we let $m(L)=\min _{j}\left|S_{j}\right|$. If $m(L)$ $>0$ then $L$ will be called a proper piecewise linear function.

Theorem 2. Let $L$ be a proper piecewise linear function on $[-\pi / 2, \pi / 2]$. Then there exists a nearly comonotone sequence $\left\{t_{n}\right\}, t_{n} \in T_{n}$, such that

$$
\left\|L-t_{n}\right\| \leq C_{7} M(L) / n \text {. }
$$

Proof. Let $-\pi / 2=x_{1}<\ldots<x_{m}=\pi / 2$ be the peaks of $L$. We will construct a sequence $\left\{t_{n}\right\}, t_{n} \in T_{n}$, satisfying (9) and such that if $0<\epsilon<1 / 2 \min \left(x_{i+1}-x_{i}\right)$ then $t_{n}$ will have the same monotonicity as $L$ on $\left[x_{i}+\epsilon, x_{i+1}-\epsilon\right], i=1,2, \ldots$, $m-1$ for all $n \geq \epsilon^{-1}\left[C_{8} M(L) / m(L)\right]^{1 / 3}$.

Let $-\pi / 2=y_{0}<y_{1}<\ldots<y_{k}=\pi / 2$ be the nodes of $L$. Let 


$$
a_{0}=1 / 2\left(S_{0}+S_{k-1}\right), \quad a_{j}=1 / 2\left(S_{j}-S_{j-1}\right), \quad j=1,2, \cdots, k-1 .
$$

Then

$$
L(x)=A+\sum_{j=0}^{k-1} a_{j}\left|x-y_{j}\right|,
$$

where $A$ is a constant. Let $t$ be defined as in Theorem $1[(6)$ and (8)] and let

$$
t_{n}(x)=A+\sum_{j=0}^{k-1} a_{j} t\left(x-y_{j}\right) .
$$

Then $t_{n} \in T_{n}$. It follows [3, p. 147] that

$$
\left|L(x)-t_{n}(x)\right| \leq \frac{C_{7}}{n} \max _{i}\left|\sum_{j=0}^{i} a_{j}\right| \leq \frac{C_{7}}{n} \max _{i}\left|S_{i}\right|=\frac{C_{7} M(L)}{n},
$$

thus establishing (9).

Now let $x \in\left[x_{i}+\epsilon, x_{i+1}-\epsilon\right]$. We assume, without loss of generality, that $L$ is increasing on $\left(x_{i}, x_{i+1}\right)$. Let $y_{q}, y_{q+1}, \ldots, y_{q}$, be those consecutive nodes (if any) of $L(x)$ which are within $\epsilon$ of $x$; i.e., such that

$$
\left|x-y_{q}\right|<\epsilon, \quad\left|x-y_{q+1}\right|<\epsilon, \quad \cdots, \quad\left|x-y_{q},\right|<\epsilon .
$$

Now, differentiating in (11), we get

$$
t_{n}^{\prime}(x)=\sum_{0}^{k-1} a_{j} r_{j}=\sum_{0}^{q-1} a_{j} r_{j}+\sum_{q}^{q^{\prime}} a_{j} r_{j}+\sum_{q^{\prime}+1}^{k-1} a_{j} r_{j},
$$

where $r_{j}$ denotes $t^{\prime}\left(x-y_{j}\right)$. For $j=q, \ldots, q^{\prime}$ we have $\left|x-y_{j}\right|<\epsilon$, hence, from Theorem 1 (iii), $r_{j}$ is decreasing as $j$ goes from $q$ to $q^{\prime}$. Also, from Theorem 1 (ii) and Theorem 1 (iv), $0 \leq r_{j} \leq 1$ for $x-y_{j} \geq 0$ and $-1 \leq r_{j} \leq 0$ for $x-y_{j} \leq$ 0 . Applying the estimates from Theorem 1 (iv) for $t^{\prime}$ to the first and third sums in (13), we get

$$
t_{n}^{\prime}(x)=\sum_{0}^{q-1} a_{j}-\sum_{0}^{q-1} a_{j} E_{j}+\sum_{q}^{q^{\prime}} a_{j}{ }^{r}{ }_{j}-\sum_{q^{\prime}+1}^{k-1} a_{j}+\sum_{q^{\prime}+1}^{k-1} a_{j} E_{j},
$$

where, from (5), we have $0 \leq E_{j} \leq C_{6} / n^{3} \epsilon^{3}$. Now, from the definition of $\left\{a_{j}\right\}$, rewriting the summands, we obtain

$$
\begin{aligned}
& \sum_{0}^{q-1} a_{j}-\sum_{q^{\prime}}^{k-1} a_{j}+\sum_{q}^{q^{\prime}} a_{j} r_{j} \\
& \quad=1 / 2\left[S_{q-1}\left(1-r_{q}\right)+\sum_{q}^{q^{\prime}-1} S_{j}\left(r_{j}-r_{j+1}\right)+S_{q^{\prime}}\left(1+r_{q^{\prime}}\right)\right] \geq m(L)
\end{aligned}
$$


by positivity of each term. Since $0 \leq E_{j} \leq C_{6} / n^{3} \epsilon^{3}$, by partial summation we obtain

$$
\left|\sum_{0}^{q-1} a_{j} E_{j}-\sum_{q^{\prime}+1}^{k-1} a_{j} E_{j}\right| \leq \frac{C_{8} M(L)}{n^{3} \epsilon^{3}} .
$$

Combining (15) and (16) in (14), we get $t^{\prime}(x) \geq m(L)-C_{8} M(L) / n^{3} \epsilon^{3}$. This quantity is $\geq 0$ for $n \geq \epsilon^{-1}\left[C_{8} M(L) / m(L)\right]^{1 / 3^{n}}$. Q.E.D.

Remark. In the proof of Theorem 2 we showed that $t_{n}$ will have the same monotonicity as $L$ on $\left[x_{i}+\epsilon, x_{i+1}-\epsilon\right], i=1,2, \ldots, m-1$, for $n \geq \epsilon^{-1}\left[C_{8} M(L) / m(L)\right]^{1 / 3}$. It is not difficult to see that $t_{n}$ and $L$ will have the same monotonicity at a point $x \in\left[x_{i}+\epsilon, x_{i+1}-\epsilon\right]$ for $n \geq \epsilon^{-1}\left[C_{8} M(L) / m(x, \epsilon, L)\right]^{1 / 3}$, where $m(x, \epsilon, L)$ denotes the minimum slope of $L$ in an $\epsilon$-neighborhood of $x$. Indeed, in view of the choice of $q, \ldots, q^{\prime},(12), S_{q}, \ldots, S_{q^{j}} \geq m(x, \epsilon, L)$; hence, when making a local estimate in (15), $m(L)$ can be replaced by $m(x, \epsilon, L)$. In particular, if $\epsilon$ is less than the distance from $x$ to the nearest node, then $n$ depends only on the slope at $x$.

A piecewise monotone function $f$ will be called proper piecewise monotone if it satisfies the following: for any $\epsilon>0$ and two successive peaks $x_{i}, x_{i+1}$ of $f$ there exists $\delta>0$ such that

$$
\left|\frac{f(x)-f(y)}{x-y}\right| \geq \delta
$$

for all $x, y$ in $\left[x_{i}+\epsilon, x_{i+1}-\epsilon\right], x \neq y$.

Theorem 3. Let $f$ be a proper piecewise monotone function on $[-\pi / 2, \pi / 2]$ such that $f \in \operatorname{Lip}_{M} 1$ (i.e., such that $f$ satisfies $\omega(f, b) \leq M b$ ). Then there is a nearly comonotone sequence $\left\{t_{n}\right\}, t_{n} \in T_{n}$, such that

$$
\left\|f-t_{n}\right\| \leq C_{9} M / n \text {. }
$$

Proof. Let $L_{n}$ be the proper piecewise linear function on $[-\pi / 2, \pi / 2]$ which has nodes at the peaks of $f$ and at the points $-\pi / 2+j \pi / n, j=0,1, \ldots$, $n$, such that $L_{n}(x)=f(x)$ at the nodes. Then $L_{n}$ and $f$ have the same peaks and the same monotonicity for all $x \in[-\pi / 2, \pi / 2]$. Also,

$$
\left\|f-L_{n}\right\| \leq M \pi / n
$$

and $M\left(L_{n}\right) \leq M / n$. Hence, by Theorem 2, there is a polynomial $t_{n} \in T_{n}$ such that

$$
\left\|L_{n}-t_{n}\right\| \leq C_{7} M / n
$$


Now $\left\|f-t_{n}\right\| \leq\left\|f-L_{n}\right\|+\left\|L_{n}-t_{n}\right\| \leq C_{9} M / n$ from (20) and (21), establishing (19).

Since $f$ is proper piecewise monotone, for any $\epsilon>0$ there exists $\delta(\epsilon)>0$ such that the slope $S_{i}$ of $L_{n}$ in $\left[y_{i}, y_{i+1}\right]$ satisfies $S_{i} \geq \delta$ whenever $\left[y_{i}, y_{i+1}\right]$ is not within $\epsilon$ of a peak. Let $n \geq \epsilon^{-1}\left[C_{8} M / \delta(\epsilon)\right]^{1 / 3}$. In view of the remark following Theorem $2, t_{n}$ has the same monotonicity as $L_{n}$ (and, hence, as $f$ ) at all points not within $\epsilon$ of a peak.

Remark. Theorems 2 and 3 were stated for the interval $[-\pi / 2, \pi / 2]$, but are easily extended to the interval $[a, a+\pi]$ for any real number $a$ via the translation $x=u+a+\pi / 2$.

Theorem 4. Let $f$ be a proper piecewise monotone function on $[a, b]$ such that $f \in \operatorname{Lip}_{M} 1$. Then there is a nearly comonotone sequence $\left\{p_{n}\right\}, p_{n} \in P_{n}$, such that $\left\|f-p_{n}\right\| \leq C_{10} M / n$.

This the orem is proved by use of the standard transformation $x=\cos \theta$, with some modifications.

Note that the class of functions for which Theorem 3 and Theorem 4 are proved includes all $f$ which have a continuous derivative that does not vanish except at the peaks. If we view monotonicity more "locally" and less "globally", we can state our results more precisely, in a sense, than we have in Theorem 3 and Theorem 4. This is done in Theorem $3^{\prime}$ and Theorem $4^{\prime}$, which are actually corollary (indeed, equivalent) to the results already established.

Theorem 3'. Let $f \in \operatorname{Lip}_{M} 1$ on $[-\pi / 2, \pi / 2]$. Then there is a sequence $\left\{t_{n}\right\}, t_{n} \in T_{n}$, such that

$$
\left\|f-t_{n}\right\|<C_{9} M / n
$$

Moreover, $f$ and $t_{n}$ will bave the same monotonicity at $x$ for all

$$
n \geq \epsilon^{-1}\left[C_{8} M / \delta(\epsilon)\right]^{1 / 3}
$$

where $\epsilon$ is the distance from $x$ to the nearest peak of $f$ and

$$
\delta(\epsilon)=\inf _{0<|b|<\epsilon}\left|\frac{f(x+b)-f(x)}{b}\right| .
$$

Theorem $4^{\prime}$. Let $f \in \operatorname{Lip}_{M} 1$ on $[a, b]$. Then there is a sequence $\left\{p_{n}\right\}, p_{n}$ $\in P_{n}$, sucb that

$$
\left\|f-p_{n}\right\| \leq C_{10} M / n \text {. }
$$

Moreover, $f$ and $p_{n}$ will have the same monotonicity at $x$ for all $n \geq$ $\epsilon^{-1}\left[C_{8} M / \delta(\epsilon)\right]^{1 / 3}$, where $\epsilon$ and $\delta(\epsilon)$ are the same as in Theorem 3'. 


\section{REFERENCES}

1. G. G. Lorentz and K. L. Zeller, Degree of approximation by monotone polynomials. I, J. Approximation Theory 1 (1968), 501-504. MR 39 \#699.

2. G. Meinardus, Approximation of functions: Theory and numerical methods, Springer, Berlin, 1964; English transl., Springer-Tracts in Natural Philosophy, vol 13, Springer-Verlag, New York, 1967. MR 31 \#547; MR 36 \#571.

3. Eli Passow, Another proof of Jackson's theorem, J. Approximation Theory 3 (1970), 146-148. MR $41 \# 7353$.

4. John A. Roulier, Monotone approximation of certain classes of functions, J. Approximation Theory 1 (1968), 319-324. MR 38 \#4875.

5. O. Shisha, Monotone approximation, Pacific J. Math. 15 (1965), 667-671. MR $32 \# 2802$.

6. W. Wolibner, Sur un polynôme d'interpolation, Colloq. Math. 2 (1951), 136-137. MR 13, 343.

7. S. W. Young, Piecewise monotone polynomial interpolation, Bull. Amer. Math. Soc. 73 (1967), 642-643. MR $35 \# 3326$.

DEPARTMENT OF MATHEMATICS, YESHIVA UNIVERSITY, NEW YORK, NEW YORK 10033

DEPARTMENT OF MATHEMATICS, TEMPLE UNIVERSITY, PHILADELPHIA, PENNSYLVANIA 19122 